\title{
A New Approach to Identify Sphincter of Oddi Dysfunction
}

\author{
Bolek L
}

Excel Diagnostics and Nuclear Oncology Center, USA

*Corresponding author: Bolek L, Excel Diagnostics and Nuclear Oncology Center, USA, E-mail: petfellow@ hotmail.com

Citation: Bolek L (2016) A New Approach to Identify Sphincter of Oddi Dysfunction. J Adv Radiol Med Image 1(1): 104. doi: 10.15744/2456-5504.1.104

Received Date: March 31, 2016 Accepted Date: July 25, 2016 Published Date: July 27, 2016

\begin{abstract}
Sphincter of Oddi dysfunction (SOD) is a known gastrointestinal disorder that has been well documented but is difficult to diagnose noninvasively.

Methods: Beginning on 08/2009 and ending on 01/2013, 57 post cholecystectomy patients with persistent abdominal pain were imaged using radionuclide hepatic scintigraphy (HIDA) for suspected SOD. When the SPECT/CT camera was installed, a dynamic phantom was developed to validate whether a 5 minute SPECT image (3-D) was able to be acquired multiple times and have adequate counts to identify the CBD and RLL.

Results: A total of 57 patients underwent hepatic scintigraphy (52 had 2-D images, 5 had 3-D images) over a period of 3 years. Using a scoring system developed at John's Hopkins University, 19 of 57 patients (33.3\%) met the criteria for SOD.

Conclusion: It was determined that SPECT/CT can function similar to a conventional and more invasive ERCP at estimating the biliary kinetics and predicting SOBP. Also it was concluded that by using SPECT/CT imaging, 3-D information about bile kinetics can be acquired and anatomical correlation is simultaneously available, thus increasing diagnostic ability and dramatically reducing processing time of a SOD study.

Keywords: Sphincter of Oddi dysfunction; SPECT/CT
\end{abstract}

\section{Introduction}

Sphincter of Oddi dysfunction (SOD) is a known gastrointestinal disorder that has been well documented but is difficult to diagnose noninvasively. It is caused by functional and mechanical abnormalities of Sphincter of Oddi (SO) [1]. Noninvasive methods to diagnose this condition have been limited either by poor sensitivity or by the complexity of processing data into meaningful results [2]. After validating a SOD protocol for two (2) years using two-dimensional (2-D) imaging, a new three dimensional (3-D) imaging protocol for diagnosing SOD was developed using a SPECT/CT camera.

\section{Materials and Methods}

Beginning on 08/2009 and ending on 01/2013, 57 post cholecystectomy patients with persistent abdominal pain were imaged using radionuclide hepatic scintigraphy (HIDA) for suspected SOD. Positive and negative results were based on a previous study as defined by the Scintigraphic Score (SS) developed and validated by actual manometric pressure measurements at Johns Hopkins University [3].

Selected patients were pretreated with an IV bolus of cholecystokinin (CCK) $(0.02 \mathrm{ug} / \mathrm{kg})$ over 3 minutes, 15 minutes prior to being injected with standard dose $(5 \mathrm{mCi})$ of disofenin, at which time continuous dynamic images were acquired for 60 minutes in the anterior planar projection (2-D). Regions of interests were drawn around the common bile duct (CBD) and right liver lobe (RLL) at intervals of 5 minutes so that time activity curves (TACs) could be generated over the course of dynamic imaging (60 mins). Using a standard spread sheet, time activity curves were plotted and were used to help calculate the Scintigraphy Score developed at Johns Hopkins University (Table 1) [3].

Sostre et al. used scoring criteria to score a total of 26 patients with suspected SOD. Patients with a total score of $<4$ (14 pts) were considered negative (-) for SOD and patients with a score $>5$ (12 pts) were positive (+) for SOD. When correlated with manometry, the study achieved $100 \%$ sensitivity and $100 \%$ specificity. Thus all $(+)$ SOD patients had an elevated basal pressure (> $40 \mathrm{mmHg}$ ), (7 pts), or demonstrated a paradoxical response to CCK with elevation of sphincter pressure (3 pts) or had normal basal pressure but showed CBD dilation and delayed contrast emptying on ERCP (2 pts). 


\begin{tabular}{|c|c|c|c|}
\hline S. No. & & Criteria & Score \\
\hline \multirow{2}{*}{1} & \multirow{2}{*}{ Peak Time } & a. Less than 10 minutes & $\mathbf{0}$ \\
\hline & & b. 10 or more minutes & 1 \\
\hline \multirow{2}{*}{2} & \multirow{2}{*}{$\begin{array}{l}\text { Time of Biliary } \\
\text { Visualization }\end{array}$} & a. Less than 15 minutes & 0 \\
\hline & & b. 15 or more minutes & 1 \\
\hline \multirow{3}{*}{3} & \multirow{3}{*}{$\begin{array}{c}\text { Prominence of } \\
\text { Biliary Tree }\end{array}$} & a. Not prominent & 0 \\
\hline & & b. Prominent major intrahepatic ducts & 1 \\
\hline & & c. Prominent small intrahepatic ducts & 2 \\
\hline \multirow{3}{*}{4} & \multirow{3}{*}{ Bowel Visualization } & a. Less than 15 minutes & 0 \\
\hline & & b. $15-30$ minutes & 1 \\
\hline & & c. More than 30 minutes & 2 \\
\hline \multirow{4}{*}{5} & \multirow{4}{*}{ CBD Emptying } & a. More than $50 \%$ & 0 \\
\hline & & b. Less than $50 \%$ & 1 \\
\hline & & c. No change & 2 \\
\hline & & d. Shows increasing activity & 3 \\
\hline \multirow{4}{*}{6} & \multirow{4}{*}{ CBD-to-Liver Ratio } & a. CBD $_{60}<$ Liver $_{60}$ & 0 \\
\hline & & b. $\mathrm{CBD}_{60}$ higher than Liver ${ }_{60}$ but lower than Liver $_{15}$ & 1 \\
\hline & & c. $\mathrm{CBD}_{60}$ higher than Liver ${ }_{60}$ and equal to Liver $_{15}$ & 2 \\
\hline & & d. $\mathrm{CBD}_{60}$ higher than both Liver ${ }_{60}$ and Liver ${ }_{15}$ & 3 \\
\hline
\end{tabular}

Table 1: Scintigraphy Score developed at Johns Hopkins University

Thus since manometry during ERCP is the gold standard for diagnosing SOD, using the above scoring criteria to identify SOD had a high likely hood of stratifying future patients into (-) and (+) SOD categories. Other investigators used imaging criteria to diagnose SOD with varying degrees of sensitivity and specificity [4].

When SPECT/CT imaging became available, a new method of imaging was developed and validated with a dynamic phantom. The standard 2-D method was converted into a 3-D dynamic imaging method in the following way; five (5) patients were pretreated with an IV bolus of CCK for 3 minutes, as described above. Dynamic imaging was started immediately after disofenin IV injection, by acquiring a rapid SPECT image every 5 minutes for a total of 60 minutes (purple arrow) (Table 2) [5]. A total of 12 SPECT images in 1 hour were acquired (blue arrow) (Table 2) [5]. Immediately afterwards a non IV contrast CT of the abdomen was acquired and fused with the SPECT images. These SPECT images were displayed sequentially to produce a dynamic movie of bile transit from the liver parenchyma into the common bile duct (CBD) and small bowel/duodenum (SB).

\begin{tabular}{|c|c|}
\hline \multicolumn{2}{|r|}{ Summary } \\
\hline Name & Description \\
\hline \multicolumn{2}{|l|}{ Patient ID } \\
\hline Study Name & CBD \\
\hline Series Name & $\mathrm{CBD}$ \\
\hline \multicolumn{2}{|l|}{ Technologist } \\
\hline \multicolumn{2}{|l|}{ Reading Physician } \\
\hline \multicolumn{2}{|l|}{ Referring Physician } \\
\hline Organ & Hepatic \\
\hline Isotope & 99m Technetium, $0.00 \mathrm{mCi}$, Disofenin \\
\hline Matrix Size & $128 \times 128$ \\
\hline Zoom & 1.23 \\
\hline Camera Preset & Tc99m-NMG \\
\hline Detectors & Both Detectors \\
\hline Orientation & Head Out \\
\hline Patient Position & Supine \\
\hline Study based setup & OFF \\
\hline Rotation Direction & $\mathrm{CW}$ \\
\hline Starting Angle & 0 \\
\hline Degrees of Rotation & 180 \\
\hline
\end{tabular}




\begin{tabular}{|c|c|}
\hline & Summary \\
\hline Name & Description \\
\hline Number of Views & 32 \\
\hline Detector Configuration & 180 \\
\hline Orbit & Circular \\
\hline Mode & Continuous \\
\hline Phase & 1 \\
\hline Pause & No \\
\hline Repeats/Pause & 12 \\
\hline Cycles/Repeat & 1 \\
\hline Time/Cycle & 5 min \\
\hline
\end{tabular}

Table 2: Rapid SPECT technique

Thus, the first 52 patients referred for SOD evaluation were imaged using planar dynamic scintigraphy in 2-D, utilizing a standard single headed gamma camera. Regions of interests (ROI) were drawn around the RLL and CBD, every 5 minutes for a total of 60 minutes to generate 12 data points on a TAC. Two TACs were generated, one for the liver and a second one for the CBD. The TACs were used to score criteria 1 and 5 of the above scoring system (Table 1) [3]. Values for criteria 2, 3, 4, and 6 were calculated by analyzing planar scintigraphic images. The ROI of the first 52 patients were determined by correlating the scintigraphic images with the gastrointestinal anatomy on a diagnostic CT or MRI scan of the abdomen to determine the most precise borders of the CBD and RLL. An abridged sample of a typical patient's ROI of the RLL and CBD (Figure 1 and 2).

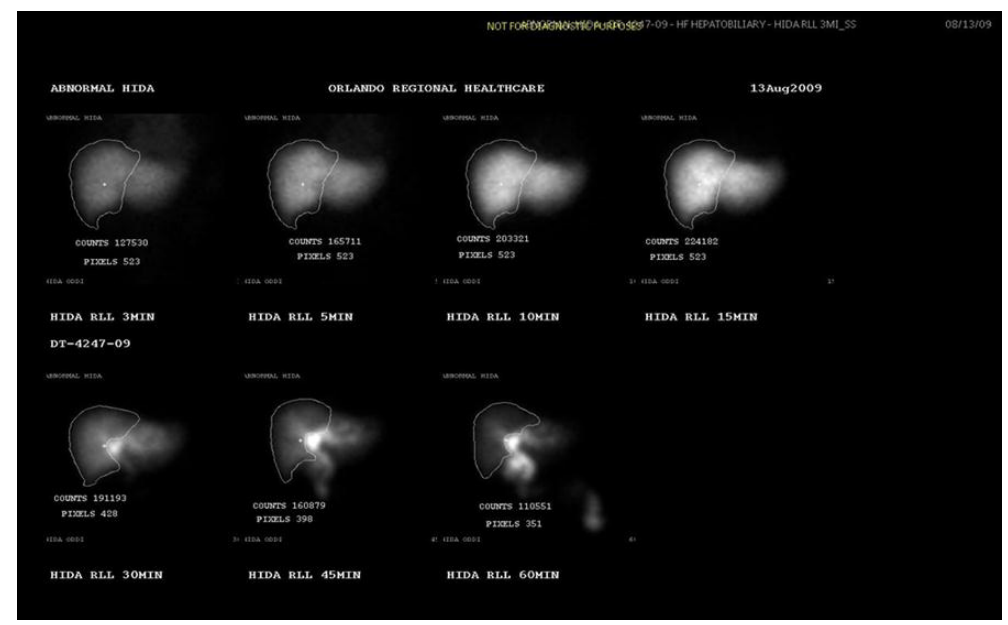

Figure 1: A ROI is manually drawn around the right liver lobe at the beginning of dynamic imaging and at selected time points throughout the 60 minutes of dynamic imaging. Note that the RLL ROI was drawn to exclude the intra-hepatic biliary tree (bottom 3 images)

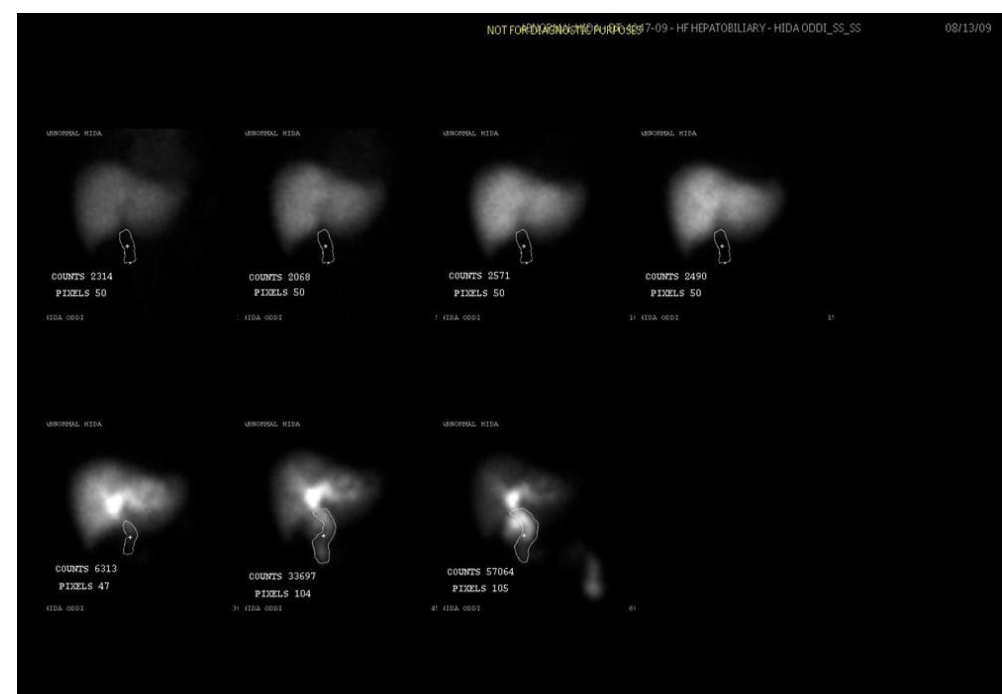

Figure 2: ROI were also manually drawn around the CBD at the beginning of planar dynamic scintigraphy and during selected time points throughout the 60 minutes of dynamic imaging 
Using the ROI counts in the right liver lobe and CBD, TACs were generated by plotting counts every 5 minutes for 60 minutes (Graph 1).

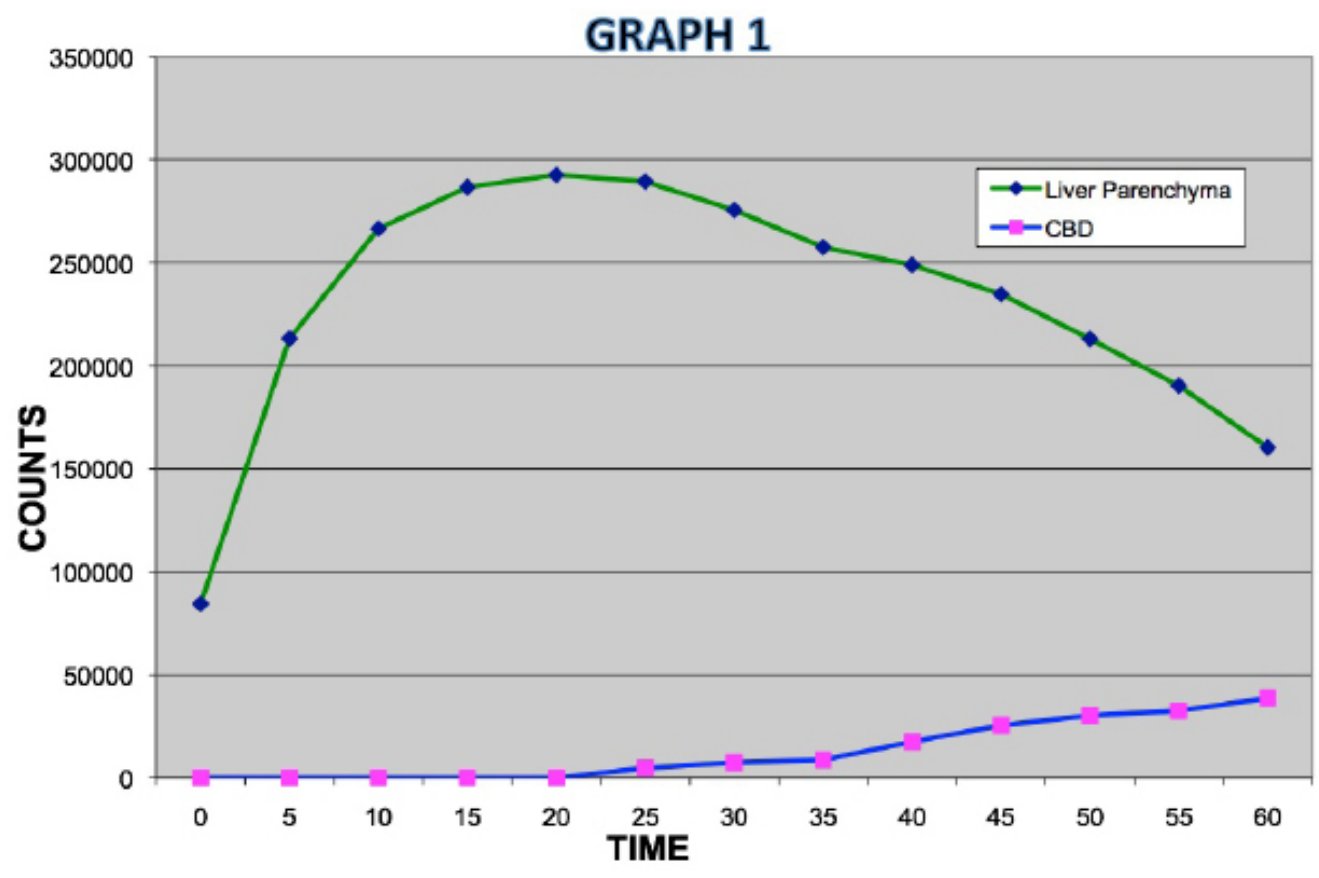

Using the above TACs, the peak hepatic time (criteria 1) was identified by looking at the graph of the RLL counts and the percent of CBD emptying (criteria 5) was calculated mathematically for each patient using the following formula:

$\left[\left(\mathrm{CBD}\right.\right.$ counts $_{\text {PEAK }}-\mathrm{CBD}$ counts $\left._{60 \mathrm{MIN}}\right) / \mathrm{CBD}$ counts $\left._{\text {PEAK }}\right] \times 100 \%=\% \mathrm{CBD}$ emptying

If there is a CONTINOUSLY RISING CURVE, the 30 min value is taken as peak CBD counts.

Prior to undergoing a HIDA scan, patients must not have received any opiate derivatives within 4 hours of the start of a hepatobiliary scan.

The following is a partial list of common opiate derivatives;

Opium Heroin

Codeine (Tylenol \#3 $3^{\circledR}$ ) Morphine (MSIR ${ }^{\oplus}$, MS Contin ${ }^{\oplus}$, Kadian ${ }^{\oplus}$ )

Hydromorphone (Dilaudid ${ }^{\circledast}$ ) Oxycodone (Percodan ${ }^{\circledast}$, Percocet $^{\circledast}$, OxyContin $^{\circledast}$ )

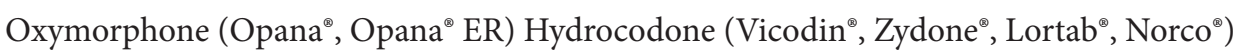

Meperidine $\left(\right.$ Demoral $\left.^{\oplus}\right)$ Fentanyl (Duragesic ${ }^{\circledast}$ )

Methadone (Dolophine ${ }^{\circledast}$ ) Tramadol (Ultram ${ }^{\circledR}$ Ultracet $^{\oplus}$ )

Naloxone $\left(\operatorname{Narcan}^{\circledast}\right)$ Tapentadol (Nucynta ${ }^{\circledast}$

Pentazocine (Talwin $\left.{ }^{\oplus}\right)$ Butorphanol

Naltrexone (ReVia ${ }^{\oplus}$, Depade ${ }^{\oplus}$, Vivitrol $\left.{ }^{\oplus}\right)$ Nalbuphine $\left(\right.$ Nubain $\left.^{\circledast}\right)$

Buprenorphine (Buprenex ${ }^{\oplus}$ ) Hydrocodeine (Synalgos ${ }^{\circledast}$ - DC)

Levorphanol

When the SPECT/CT camera was installed, a dynamic phantom was developed to validate whether a 5 minute SPECT image was able to be acquired multiple times and have adequate counts to identify the CBD and RLL. It was placed inside the gantry of a dual headed SPECT/CT (6 slice) camera [5] while the radioactive fluid (5 $\mathrm{mCi}$ of Tc99m saline) was manually drawn from the top of the phantom (liver reservoir) through plastic tubing $(\sim 1 \mathrm{~cm}$ in diameter) simulating a CBD and into a bottom reservoir simulating the duodenum and small bowel (SB) to mimic bile flow in a patient (Figure 3 and 4). 


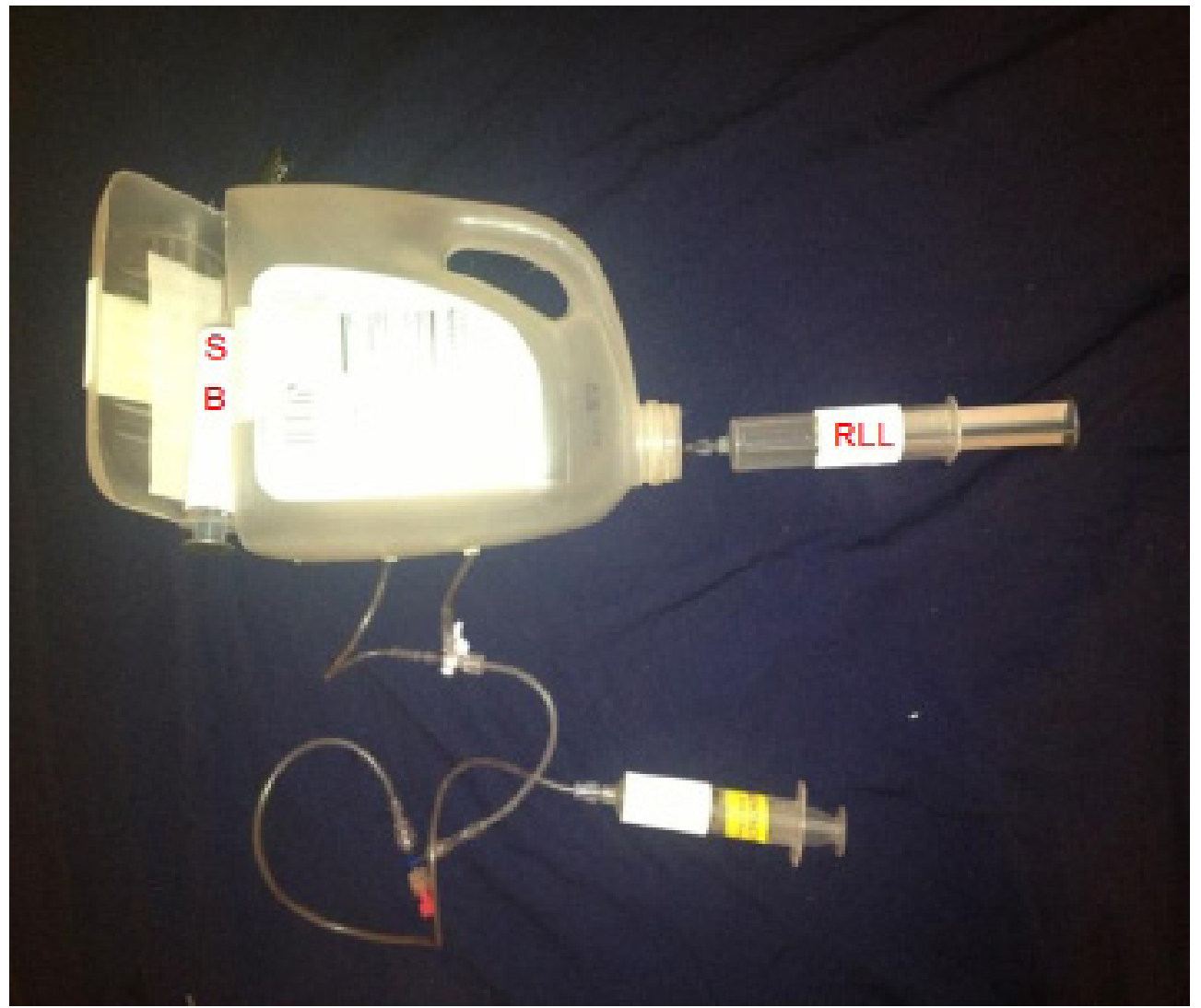

Figure 3: The top syringe was filled with Tc99m saline to simulate the RLL(Right Liver Lobe). It is connected to the bottom syringe (which simulates the duodenum/SB(Small Bowel)) by plastic tubing that simulates the CBD. The external syringe was used to draw the saline manually from the top syringe to the bottom syringe to simulate bile flow

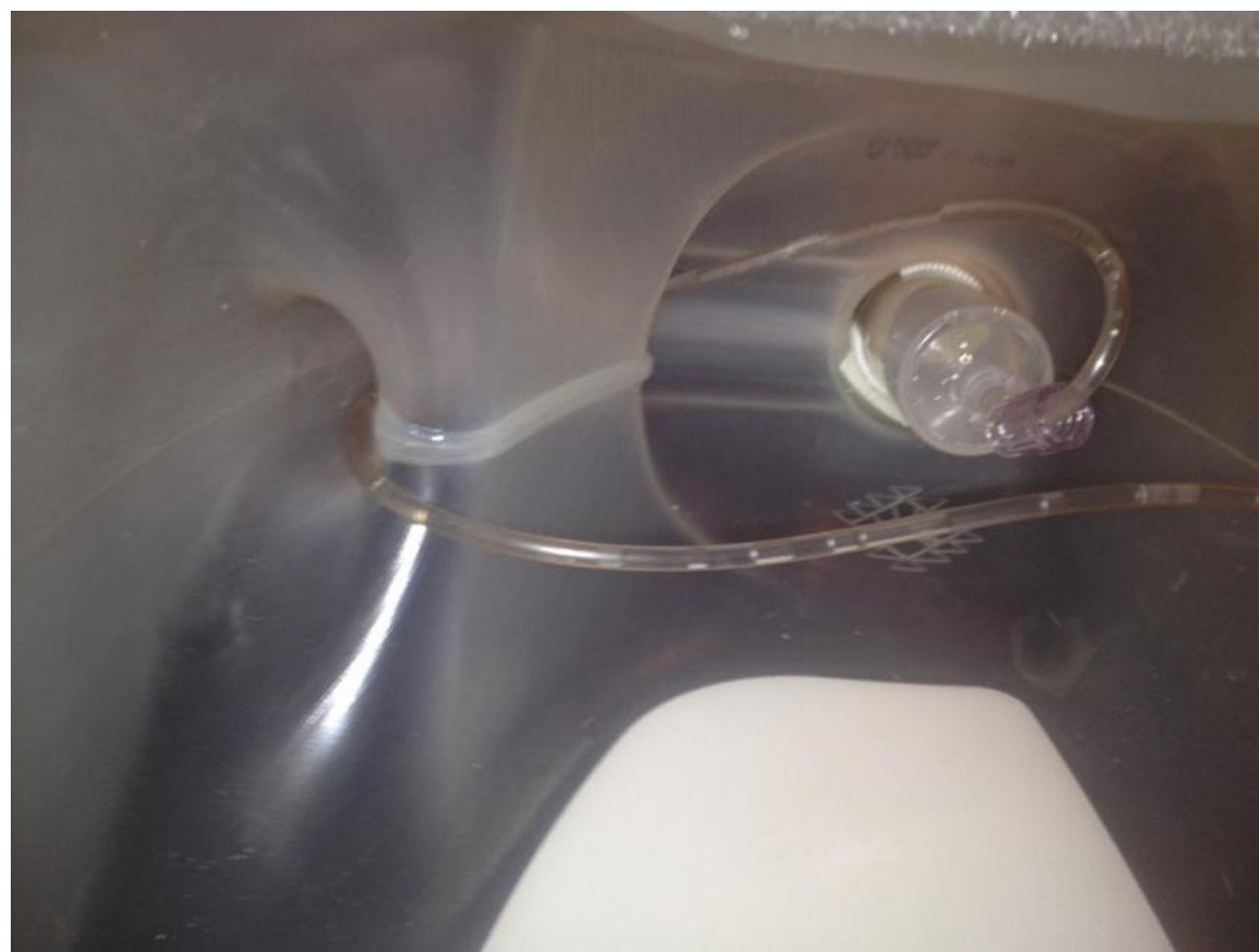

Figure 4: The inside of the phantom shows Tc99m saline flowing from the top syringe into the plastic tubing which simulates the CBD. Because actual patients often have tortuous CBDs, this plastic tubing was also bent in multiple directions to simulate the unpredictable position of the CBD

The following illustrations show the path of Tc99m saline flow through the phantom which was inside the SPECT/CT camera gantry, as the SPECT images were being acquired every 5 minutes (Figure 5, 6 and 7). 


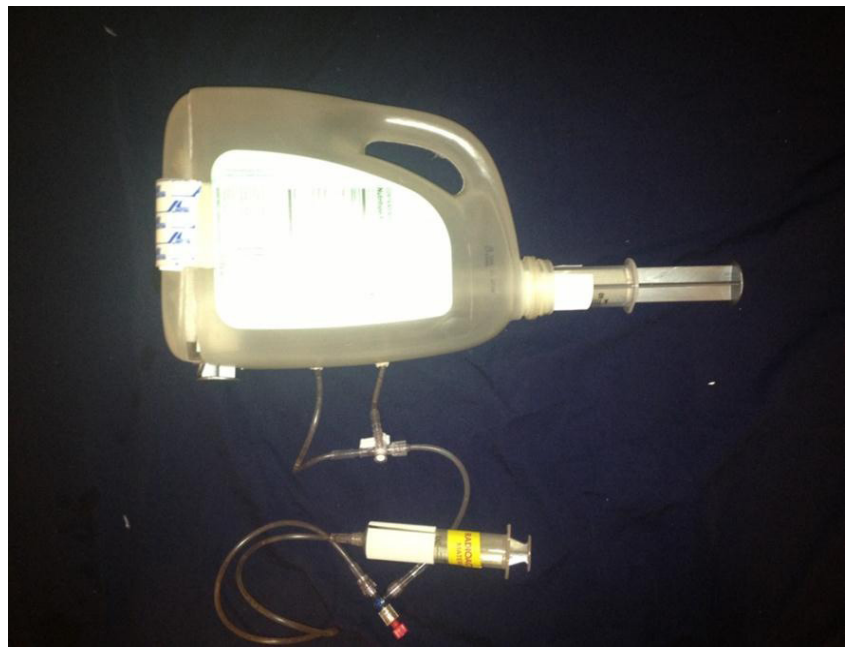

Figure 5: At the start of rapid SPECT imaging, the top syringe is full of Tc99m saline (plunger is extended). The fluid was drawn out manually form the top syringe while the dual camera heads were rotated $180^{\circ} / 5 \mathrm{~min}$

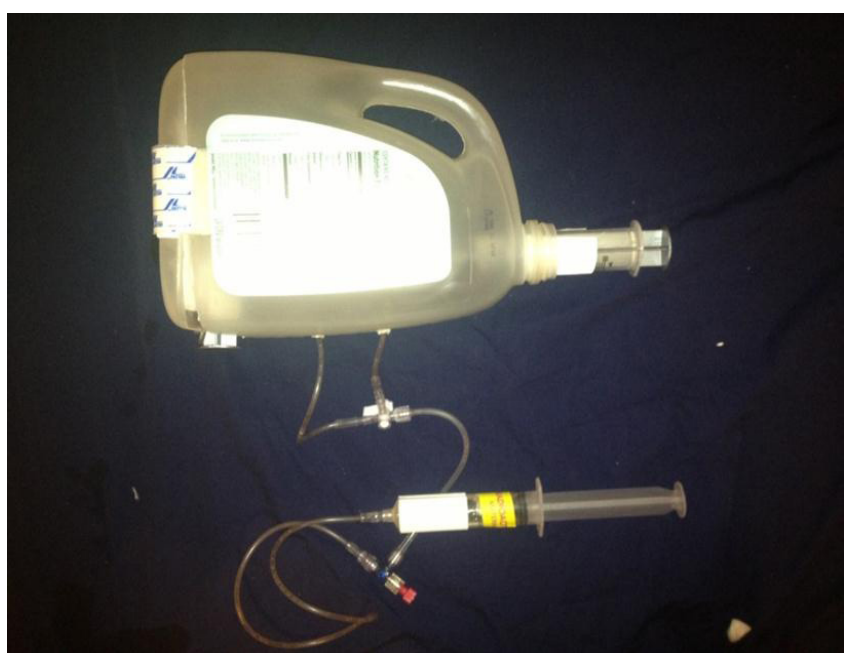

Figure 6: After a 5 min period of time all the Tc99m saline was drawn from the top syringe (RLL) (the plunger is depressed) into the external syringe (the plunger is extended, due to saline in the barrel), while the dual headed camera rotated and a rapid SPECT image $(5 \mathrm{~min})$ was acquired

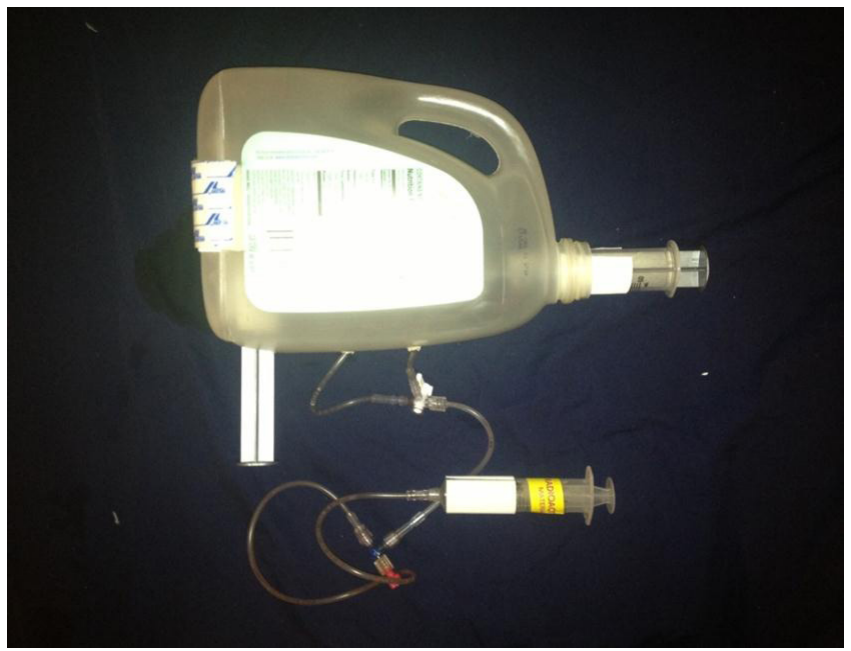

Figure 7: The stop cock on the external tubing was turned so that the Tc99m saline could be slowly pushed form the external syringe to the bottom syringe, thus simulating the flow of bile form the liver through the CBD and into the duodenum/SB (the bottom syringe in this phantom) while SPECT imaging was acquired for another $5 \mathrm{~min}$. The external syringe was completely emptied (plunger is depressed) once the stop cock was turned while SPECT imaging was continuously acquired. Tc99m saline flowed through the plastic tubing (CBD) into the bottom syringe (simulated duodenum/SB) until it was full (extended plunger). Once the phantom images validated that a rapid SPECT image could be acquired with enough counts to identify the RLL and CBD, a total of 5 patients were imaged using the SPECT/CT protocol (patients). 


\section{Results and Discussion}

A total of 57 patients underwent hepatic scintigraphy (52 Add criteria 1 to 6 for the Scintigraphic Score had 2-D images, 5 had 3-D images) over a period of 3 years. Using a scoring system developed at John's Hopkins University [3], 19 of 57 patients (33.3\%) met the criteria for SOD. Images as well as time activity curves clearly showed significant differences in radiotracer retention in the biliary tree and peak hepatic times for patients that were positive and negative for SOD and correlated well with the gastroenterologists' referral pattern (Table 3 and 4).

\begin{tabular}{|c|c|c|c|c|c|c|c|}
\hline \multicolumn{8}{|c|}{ CRITERIA } \\
\hline \multicolumn{8}{|c|}{ Add Criteria 1 to 6 for the Scintigraphic Score } \\
\hline Patient & 1 & 2 & 3 & 4 & 5 & 6 & SS \\
\hline $\mathrm{S}, \mathrm{P}$ & 1 & 0 & 0 & 0 & 0 & 1 & $=2$ \\
\hline $\mathrm{S}, \mathrm{L}$ & 0 & 0 & 1 & 0 & 0 & 0 & $=1$ \\
\hline $\mathrm{D}, \mathrm{A}$ & 1 & 0 & 1 & 0 & 0 & 1 & $=3$ \\
\hline G,C & 1 & 0 & 1 & 0 & 0 & 1 & $=3$ \\
\hline C,A & 1 & 0 & 0 & 0 & 0 & 1 & $=2$ \\
\hline G,M & 1 & 0 & 1 & 1 & 1 & 0 & $=4$ \\
\hline G,J & 1 & 0 & 0 & 1 & 1 & 0 & $=3$ \\
\hline $\mathrm{C}, \mathrm{M}$ & 0 & 0 & 0 & 0 & 0 & 0 & $=0$ \\
\hline $\mathbf{B}, \mathbf{A}$ & 1 & 0 & 1 & 0 & 1 & 1 & $=4$ \\
\hline $\mathrm{P}, \mathrm{L}$ & 1 & 0 & 0 & 0 & 0 & 1 & $=2$ \\
\hline T,M & 1 & 0 & 0 & 1 & 0 & 2 & $=4$ \\
\hline C,I & 0 & 0 & 1 & 0 & 1 & 0 & $=2$ \\
\hline $\mathrm{H}, \mathrm{S}$ & 0 & 0 & 0 & 0 & 0 & 1 & $=1$ \\
\hline $\mathbf{M}, \mathbf{H}$ & 0 & 0 & 1 & 0 & 1 & 0 & $=2$ \\
\hline $\mathrm{H}, \mathbf{M}$ & 1 & 0 & 1 & 0 & 0 & 1 & $=3$ \\
\hline H,E & 1 & 0 & 0 & 0 & 0 & 0 & $=1$ \\
\hline P,J & 0 & 0 & 1 & 0 & 0 & 1 & $=2$ \\
\hline $\mathbf{C}, \mathbf{R}$ & 1 & 0 & 0 & 0 & 0 & 3 & $=4$ \\
\hline $\mathrm{M}, \mathrm{E}$ & 1 & 0 & 0 & 0 & 1 & 1 & $=3$ \\
\hline H,J & 0 & 0 & 0 & 0 & 0 & 1 & $=1$ \\
\hline $\mathrm{D}, \mathbf{R}$ & 0 & 0 & 0 & 0 & 1 & 2 & $=3$ \\
\hline $\mathrm{J}, \mathrm{C}$ & 0 & 0 & 0 & 0 & 1 & 1 & $=2$ \\
\hline $\mathbf{M}, \mathbf{N}$ & 1 & 0 & 0 & 0 & 0 & 0 & $=1$ \\
\hline $\mathrm{K}, \mathrm{J}$ & 1 & 0 & 0 & 0 & 1 & 1 & $=3$ \\
\hline $\mathrm{A}, \mathrm{E}$ & 1 & 0 & 0 & 1 & 0 & 0 & $=2$ \\
\hline $\mathrm{W}, \mathrm{L}$ & 1 & 0 & 0 & 0 & 0 & 1 & $=2$ \\
\hline $\mathbf{L}, \mathbf{T}$ & 1 & 0 & 0 & 0 & 1 & 1 & $=3$ \\
\hline B,A & 0 & 0 & 2 & 0 & 0 & 1 & $=3$ \\
\hline $\mathrm{A}, \mathrm{C}$ & 1 & 0 & 1 & 0 & 0 & 0 & $=2$ \\
\hline P,I & 1 & 0 & 0 & 0 & 0 & 1 & $=2$ \\
\hline $\mathbf{M}, \mathbf{R}$ & 1 & 0 & 1 & 0 & 0 & 0 & $=2$ \\
\hline T,D & 1 & 1 & 1 & 0 & 0 & 1 & $=4$ \\
\hline $\mathbf{L}, \mathbf{R}$ & 0 & 0 & 0 & 0 & 0 & 0 & $=0$ \\
\hline $\mathrm{H}, \mathrm{J}$ & 1 & 0 & 0 & 0 & 0 & 0 & $=1$ \\
\hline$H, E$ & 1 & 0 & 0 & 1 & 0 & 1 & $=3$ \\
\hline$R, H$ & 1 & 0 & 0 & 0 & 0 & 1 & $=2$ \\
\hline$K, L$ & 1 & 0 & 0 & 1 & 1 & 1 & $=4$ \\
\hline$B, L$ & 0 & 0 & 1 & 0 & 1 & 1 & $=3$ \\
\hline
\end{tabular}

Table 3: SOD (-) Patients 
This cohort of patients was evaluated using the John's Hopkin's Scintigraphic score among 4 different interpreting physicians. There was very little inter-observer variability because each study was processed consistently. Although there was slight differences between the scoring among the physicians (+/- 1 point), the sum score always resulted in $100 \%$ agreement on whether or not the patient was SOD $(+)$ or $(-)$. There are a total of 6 diagnostic criteria, and each physician had to go through the same process of scrutinizing the dynamic images. Since each criteria uses a rising scale, it did not matter if there was exact agreement on each criteria's score, as long as sum total used to diagnose SOD, was $<4$ or $>5$. This scale is a measure of bile kinetics through the biliary system. It starts at the beginning of the biliary system (liver) and tracks the bile flow to the end of the biliary pathway (duodenum). Furthermore criteria 1 and 5 are absolute numbers which are not subjective and all 4 interpreting physicians must score them the same, based on the graphical data.

\begin{tabular}{|c|c|c|c|c|c|c|c|}
\hline \multicolumn{7}{|c|}{ CRITERIA } \\
\hline \multicolumn{7}{|c|}{ Add Criteria $\mathbf{1}$ to $\mathbf{6}$ for the Scintigraphic Score } \\
\hline Patient & $\mathbf{1}$ & $\mathbf{2}$ & $\mathbf{3}$ & $\mathbf{4}$ & $\mathbf{5}$ & $\mathbf{6}$ & SS \\
\hline D,C & 1 & 0 & 1 & 1 & 2 & 2 & $=\mathbf{7}$ \\
\hline M,A & 0 & 0 & 1 & 1 & 1 & 3 & $=\mathbf{6}$ \\
\hline K,B & 1 & 1 & 0 & 1 & 3 & 3 & $=\mathbf{9}$ \\
\hline O,M & 1 & 0 & 0 & 0 & 1 & 3 & $=\mathbf{5}$ \\
\hline R,J & 0 & 0 & 1 & 1 & 1 & 3 & $=\mathbf{6}$ \\
\hline W,W & 0 & 0 & 1 & 1 & 1 & 3 & $=\mathbf{6}$ \\
\hline V,D & 1 & 1 & 2 & 1 & 1 & 3 & $=\mathbf{9}$ \\
\hline C,C & 1 & 1 & 1 & 2 & 3 & 2 & $=\mathbf{1 0}$ \\
\hline M,L & 1 & 0 & 1 & 0 & 1 & 2 & $=\mathbf{5}$ \\
\hline H,Q & 1 & 0 & 1 & 1 & 1 & 2 & $=\mathbf{6}$ \\
\hline S,T & 1 & 1 & 1 & 1 & 1 & 3 & $=\mathbf{8}$ \\
\hline P,D & 1 & 0 & 0 & 1 & 1 & 3 & $=\mathbf{6}$ \\
\hline W,T & 1 & 1 & 0 & 0 & 1 & 2 & $=\mathbf{5}$ \\
\hline G,A & 1 & 1 & 1 & 1 & 1 & 3 & $=\mathbf{8}$ \\
\hline W,B & 1 & 1 & 1 & 1 & 1 & 3 & $=\mathbf{8}$ \\
\hline H,R & 1 & 0 & 1 & 0 & 1 & 2 & $=\mathbf{5}$ \\
\hline G,E & 1 & 1 & 0 & 1 & 1 & 2 & $=\mathbf{6}$ \\
\hline O,S & 1 & 0 & 0 & 1 & 2 & 1 & $=\mathbf{5}$ \\
\hline S,C & 1 & 1 & 0 & 1 & 1 & 3 & $=\mathbf{7}$ \\
\hline & & & & & & & \\
\hline
\end{tabular}

Table 4: SOD (+) Patients

As with previous studies, patients with obvious SOD had slower biliary transit through the biliary system. The higher the score, the greater retention of radiotracer in the biliary system. For example, patients with the highest scintigraphic score had the greatest retention of radiotracer in the $\mathrm{CDB}$ (criteria 5 and 6) and the longest time to bowel visualization (criteria 4).

Previous studies were designed to look only at one or two similar criteria. For example, one prior investigators looked at hepatic hilum to duodenum transit time (HHDT) as well as scored each patient using the John's Hopkin's SS and reported a lower sensitivity (25-38\%) and specificity (86-89\%) for identifying SOD [6]. However the study design was altered as CCK-8 was infused at 20 ng/ $\mathrm{kg} /$ body weight over 45 minutes, starting 15 minutes before quantitative hepatobiliary scintigraphy (QHBS), and the infusion was continued during the first 30 minutes of the QHBS study.

This likely resulted in the CCK receptors being continuously stimulated as the concentration of this hormone in the blood was high enough to uniformly accelerate transpapillary bile flow, thus masking basal bile flow differences in SOD patients. In most SOD patients with an elevated sphincter of Oddi basal pressure (SOBP), CCK-8 induces a significant pressure drop, as demonstrated by Hogan and Geenen [7]. Thus it can be inferred that this cohort of patients with clinical SOD symptoms had a normal response to CCK, due to the long infusion time. Thus only the few (+) SOD patients with the highest elevated SOBP (well above $40 \mathrm{mmHg}$ ) were identified in this study which had poor sensitivity and specificity (13\% and 95\%) for a HDTT > 9 mins, because CCK stimulation was not able to lower their SOBP $<40 \mathrm{mmHg}$.

Furthermore this cohort of patients were not adequately evaluated for biliary dyskinesia (a paradoxical response to CCK), as a short bolus of CCK was never given. A bolus of CCK-8 is essential for causing the SO to spasm as demonstrated by manometry when $1 \mathrm{U} / \mathrm{kg} /$ body weight was infused IV over 30 seconds [8]. However this is only one criteria for diagnosis of SOD on ERCP/ manometry and it is a relatively rare phenomena occurring in less than $25 \%$ of all SOD patients [9]. 
In a second study, a group of patients with abdominal pain underwent biliary scintigraphy without CCK stimulation to measure the HHDT, which was significantly elevated (>9 min) in all patients with maximal SOBP $>40 \mathrm{mmHg}$ [10]. This investigator achieved a higher sensitivity of $68.18 \%$ when using HHDT $>9$ min to identify SOD, as some patients with elevated HHDT had normal SO pressure $(<40 \mathrm{mmHg})(7 / 15 \mathrm{pts})$. His specificity was $56 \%$ when the HHDT was $>9$ minutes. This study was more predictive of SOD and confirms earlier reports that many patients with SOD symptoms have elevated SOBP and are less likely to excrete bile through the CBD into the small bowel in a normal period of time [11]. Their symptoms improved after endoscopic sphincterotomy by increasing bile flow through the biliary tree.

A third investigator examined two criteria $\left(\mathrm{T}_{\max }\right.$ and \% excretion ${ }_{45 \& 60 \mathrm{MIN}}$ of bile) but used morphine provocation of the $\mathrm{SO}(0.04 \mathrm{mg} /$ $\mathrm{kg}$ ). By combining two criteria, he achieved a sensitivity and specificity of $83 \%$ and $81 \%$ respectively for discriminating between patients with normal and abnormal SOBP (> $40 \mathrm{mmHg})$ [12]. It is well documented that morphine increases the contraction and frequency of the SO in healthy people [13]. However this study showed that patients with SOD (SOBP $>40 \mathrm{mmHg})$ exhibit a hypersensitivity/enhanced response to morphine as their SOBP rose to higher levels than in healthy people and decreasing the excretion of radioactive bile at 45 and especially 60 minutes of imaging.

The above findings indicate that SOD is a vague but multifactorial problem. As such, it is necessary to evaluate the biliary system at multiple levels. In 2000, it was demonstrated that hepatobiliary imaging must have multiple criteria that need to be analyzed before SOD can be diagnosed. In one study, patients were evaluated for HHDT, duodenal appearance time (DAT), CBD T max $_{\text {and }}$ $\mathrm{T}_{1 / 2}$ of bile [14]. Laszlo et al. demonstrated that SOD $(+)$ patients had a statistically significant linear correlation between a SOBP $>$ $40 \mathrm{mmHg}$ and longer HHDT, with a sensitivity of $89 \%$ for identifying (+) SOD patients. When two additional criteria were added (CBD $\mathrm{T}_{\max }$ and $\mathrm{T}_{1 / 2}$ of bile), sensitivity and specificity increased to $100 \%$ [14]. CCK-8 did not induce a paradoxical SO response in the 20 patients. However CCK was infused at 60 minutes of imaging at a lower concentration $(5 \mathrm{ng} / \mathrm{kg})$ over a longer period of time (10 $\mathrm{min})$ when compared with Sostre's protocol $(20 \mathrm{ng} / \mathrm{kg}=0.02 \mathrm{ug} / \mathrm{kg}, 3 \mathrm{~min})$ [3].

Thus it is not surprising that Sostre et al. showed a 100\% sensitivity and specificity of diagnosing SOD, as 6 criteria were used to analyze the biliary system of patients with abdominal pain. Such stringent criteria helps address at least two phenomena seen in SOD (SOBP > $40 \mathrm{mmHg}$ and paradoxical response to CCK). It appears there can be (+) SOD patients with just elevated SOBP resulting in delayed clearance of bile. When IV CCK-8 is rapidly infused 15 minutes prior to 1 hour of dynamic imaging in Sostre's protocol, the SOBP may not drop to a normal range to allow bile to drain rapidly, likely because CCK-A receptors at the SO are sparsely expressed in (+) SOD patients. Also the half time of exogenous CCK- 8 activity in the body is approximately 2.5 minutes [15]; thus CCK's effect in (+) SOD patients is minimized at the SO when dynamic imaging is started 15 minutes later.

Furthermore, the Sostre's protocol also identifies (+) SOD patients that have a paradoxical response to CCK. These patients likely are hypersensitive to CCK before imaging is started, and there is sufficient time for the CCK to stimulate the usually dormant contractile CCK receptors at the SO [16]. Thus the author postulates that in (+) SOD patients with paradoxical response to CCK, there is increased expression of CCK-B receptors at the sphincter of Oddi. In other studies CCK-B receptors have been shown to behave differently than CCK-A receptors. Gonzelez et al. showed that in the human esophagus, the contractile effect of CCK-8 was not significantly altered when the CCK-B receptor antagonists were present in the organ bath at higher concentrations, but the CCK-8-induced contraction was blocked by the CCK-A receptor antagonists [17]. Thus these CCK-B receptors likely are also present on the SO, and by the time imaging is started (15 min post CCK injection) the SO is already contracting and increasing the intra-biliary pressure. Although by 60 minutes of imaging, CCK is no longer detectable in human blood, it likely is still stimulating the CCK-B receptors which maybe overexpressed in some (+) SOD patients. In healthy individuals, CCK-B receptor are sparsely found on the SO and maintain a much higher threshold for CCK-8 than the relaxation (CCK-A) receptors.

This concept was very clearly demonstrated in the current study with one specific patient (C,C). This individual (C,C) had two HIDA scans, one with and one without CCK stimulation. The scintigraphic score was significantly lower without CCK stimulation $(S S=3)$. The only variable added to the second HIDA scan was CCK-8, and it raised the final SS to 10. Thus, this patient exhibited true SO contraction, raising her normal SOBP $(<40 \mathrm{mmHg})$ for an hour and slowing the bile clearance, despite the short CCK half-life of 2.5 minutes. Therefore it can be concluded that this patient had a sphincter of Oddi that behaved paradoxically to low levels of CCK, likely due to overexpression of stimulatory receptors such as CCK-B receptors, not usually found in high concentrations in healthy individuals. Finally, some (+) SOD patients simply have a dilated CBD due to years of elevated SOBP caused by a combination of the above two phenomena, resulting in slow and delayed bile clearance.

It becomes clear that although there are many ways to evaluate SOD noninvasively, Sosteri's protocol had correlation with manometry which yielded the most accurate results (100\% sensitivity and specificity). However data collection to reproduce this study is very demanding. The average processing time for each study was $>30$ minutes. For patients with complicated anatomy (tortuous CBD, gastric bypass, Whipple surgery etc.) the processing took even longer ( $>45 \mathrm{~min})$. In these patients, the ROI had to be compared with a diagnostic CT or MRI of the abdomen so that the beginning of the CBD (at the surgical clips/cholecystectomy site) could be identified and the end of the CBD (at the junction of the duodenum) could be found. Thus ROI often had to be redrawn and adjusted so there was as little possible overlap between the CBD and duodenum and hepatic hilum. Because the resolution of a typical planar gamma camera is lower than that of a CT and MRI scan, identifying structures $<2 \mathrm{~cm}$ is often difficult. 
The task of identifying the biliary tree on 2-D scintigraphy is challenging since the upper limit of normal diameter of a common bile duct (CBD) on ultra sound (US) is $6-8 \mathrm{~mm}$ (lumen) and that of common hepatic duct (CHD) diameter is $6 \mathrm{~mm}$. However, on CT scan it is more common to accept a value of $8-10 \mathrm{~mm}$ for CBD [18]. This is because CT visualizes mid to distal CBD and the measurement includes the duct wall. On the other hand, ERCP and cholangiogram may reveal a duct diameter up to $10.6 \mathrm{~mm}$ because of magnification of the cholangiogram and may also reflect ductal distension from contrast injection [19]. Measurements may also vary according to the age of the individual. A trend of increasing CBD diameter has been noticed in older individuals. In general, one may add $1 \mathrm{~mm}$ to the upper limit of normal CBD for each decade of life after 60 years [18].

Thus despite even with the most generous estimations of normal CBD diameter, a conventional gamma camera is not able to resolve clear transitions between the liver and CBD or the CBD and duodenum. The overlap of bowel and CBD will often increase during dynamic imaging as there is peristalsis of the bowel loops especially when high concentrations of CCK is given to the patient [15]. These limitations are difficult to overcome on traditional 2-D cholescintigraphy, as there is a significant uncertainty as to the exact anatomical location of the three organs of interests (liver, CBD, and duodenum) [11]. To complicate matters even more, 2-D processing must be done manually by drawing regions of interest around the RLL and CBD, EVERY 5 MINUTES, FOR 60 MINUTES, for a total of 12 times. This is especially critical for the CBD as this ROI often is difficult to identify early into the study ( $<15 \mathrm{~min})$ as there is no bile accumulation in the structure. Overlapping the CBD ROI with the hepatic hilum, will give artificially high results in the beginning of the TAC. Also, during late imaging ( $>30 \mathrm{~min}$ ) loops of bowel in 2-D become filled with bile and overlap with the CBD, due to peristalsis. The technologist must be careful to avoid including activity from the loop of bowel in the CBD ROI. Lastly many patients with abdominal pain move from frame to frame, as this is a lengthy study, and CCK infusion often induces shooting pain in the right upper quadrant. Each frame must be scrutinized to ensure that there is no overlap between the ROIs. Although quantitative hepatobiliary scintigraphy has been able to identify SOD, the above technical challenges exemplify why it has not been routinely offered to gastroenterologists by a nuclear medicine physician.

With the advent of SPECT/CT cameras, most anatomical structures now can be evaluated with pinpoint precision. Because the CT component can outline the anatomy to sub centimeter levels, the author theorized that it can play a vital role in imaging radionuclide movement through the biliary system (Figure 8) [19]. Essentially SPECT/CT can function similar to a conventional and more invasive ERCP (Figure 9) at estimating the biliary kinetics and predicting SOBP.

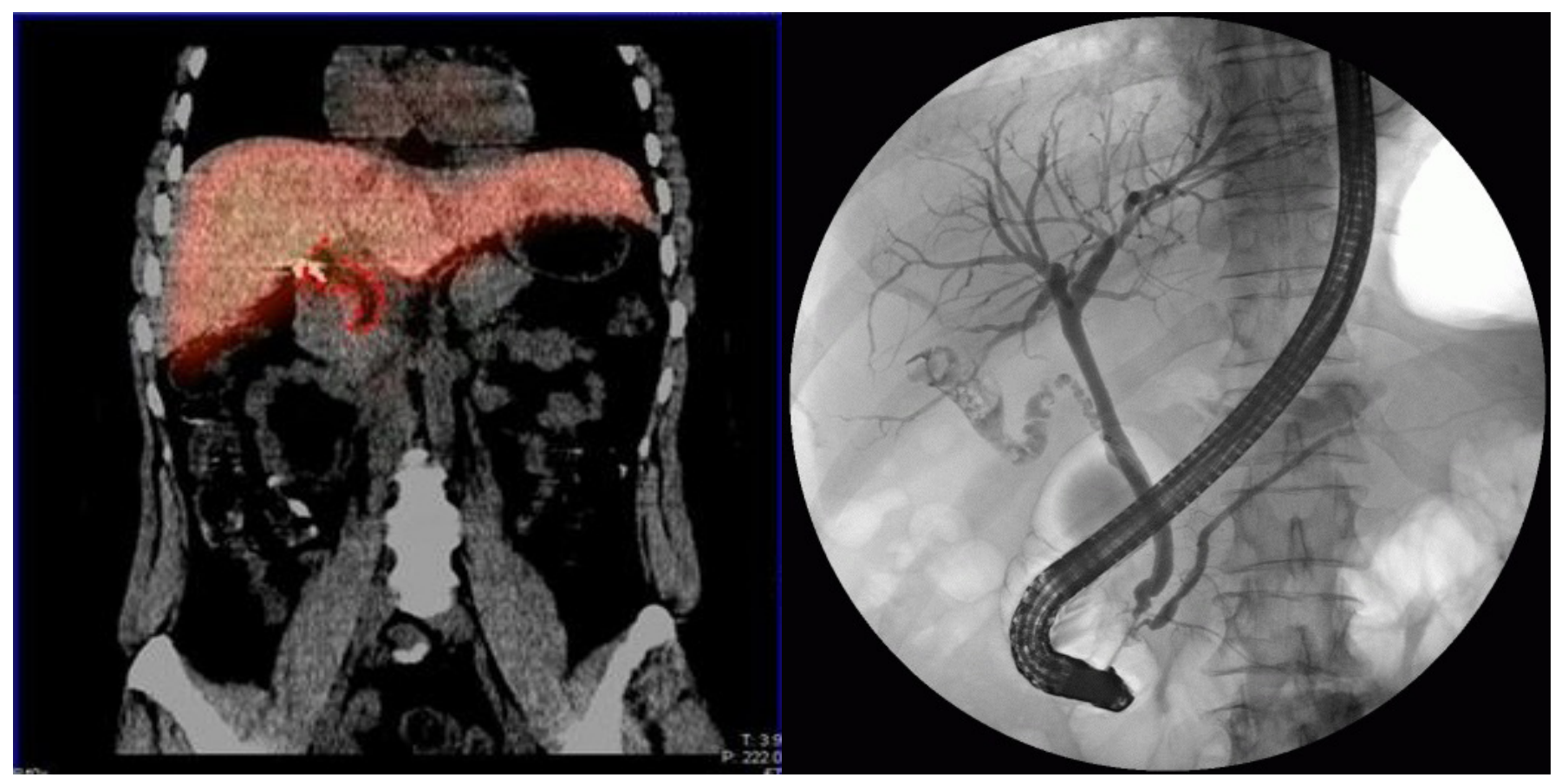

Figure 8: Note the metallic clip at the cholecystectomy site

Figure 9: ERCP in a patient shows the biliary tree anatomy SPECT/CT imaging proved far superior at identifying the regions of interest (RLL and CBD) and separating them form the loops of small bowel (duodenum) when compared with 2-D imaging. Furthermore the processing time for 3-D imaging was significantly faster ( $>50 \%)$ when compared with 2-D processing time, as time activity curves are generated automatically after one region of interest was drawn for the CBD and RLL. Figure 8 shows that 3-D dynamic SPECT/CT SOD study with a ROI around the CBD has a more precise correlation with an ERCP (Figure 9) at estimating the borders of the complex biliary tree anatomy then 2-D cholescintigraphy. 
Routine SPECT/CT images currently are performed statically (one 3-D SPECT image at one time point, such as when a 60 min nuclear 3-D study is fused to one anatomical structure, i.e. liver) to localize radioactivity in an organ. This is the first time (to the authors knowledge) it has been shown that consecutive SPECT images can be acquired to perform a dynamic study of radiotracer movement (kinetics) through the biliary system. There was no increase in the acquisition time for 3-D images when compared with 2-D images. The interpretation confidence of 3-D images was significantly improved as anatomical identification of the vital organs is much easier using hybrid SPECT/CT imaging (Figure 10) [19].

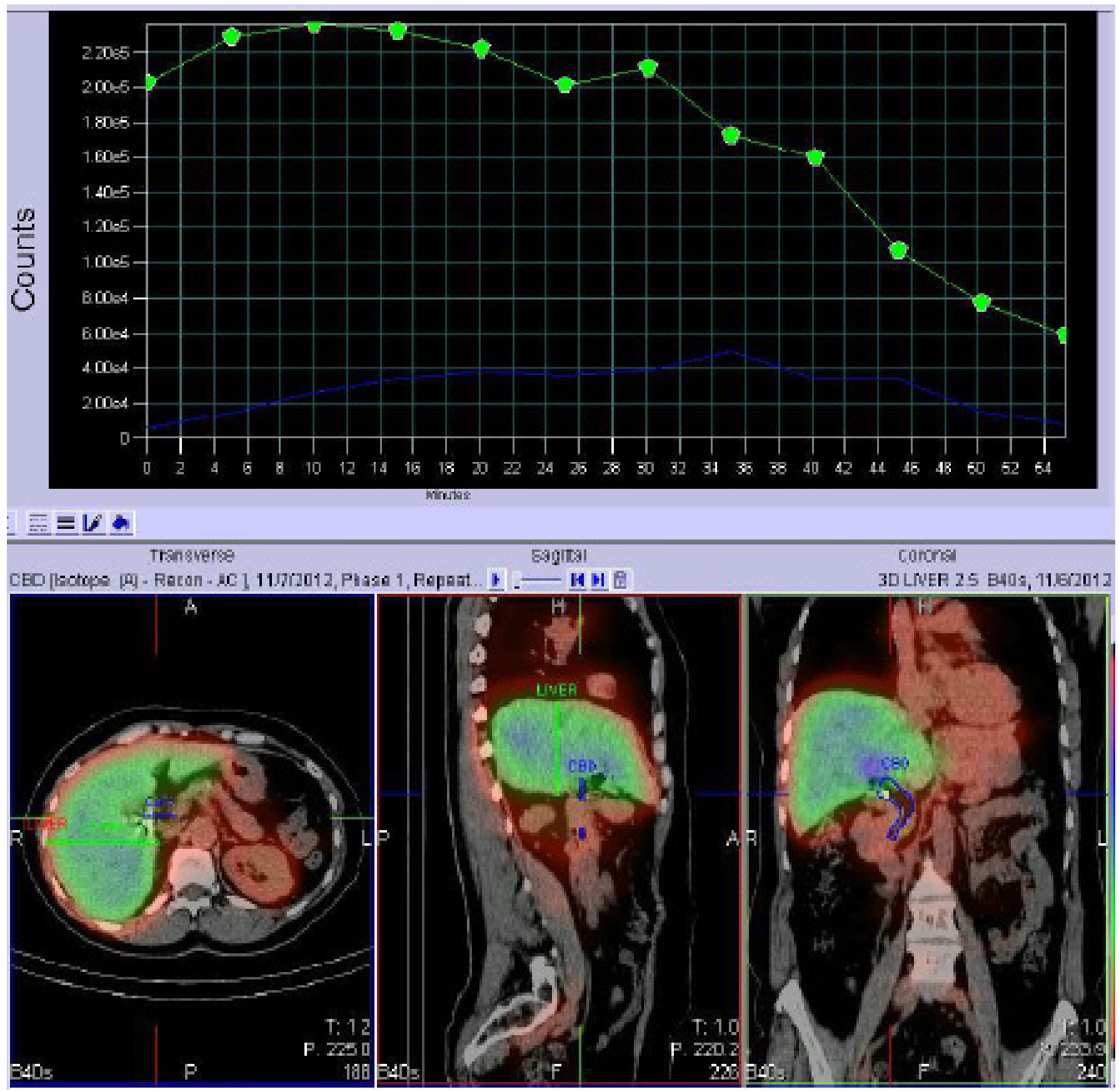

Figure 10: A (-) SOD patient with normal/rapid clearance of bile from the liver and CBD 
Furthermore in pts with $(+)$ SOD, it was much easier to identify the vital structures of the biliary treee and to track the bilary kinetics over time, as shown in the following SOD SPECT/CT study (Figure 11) [19].

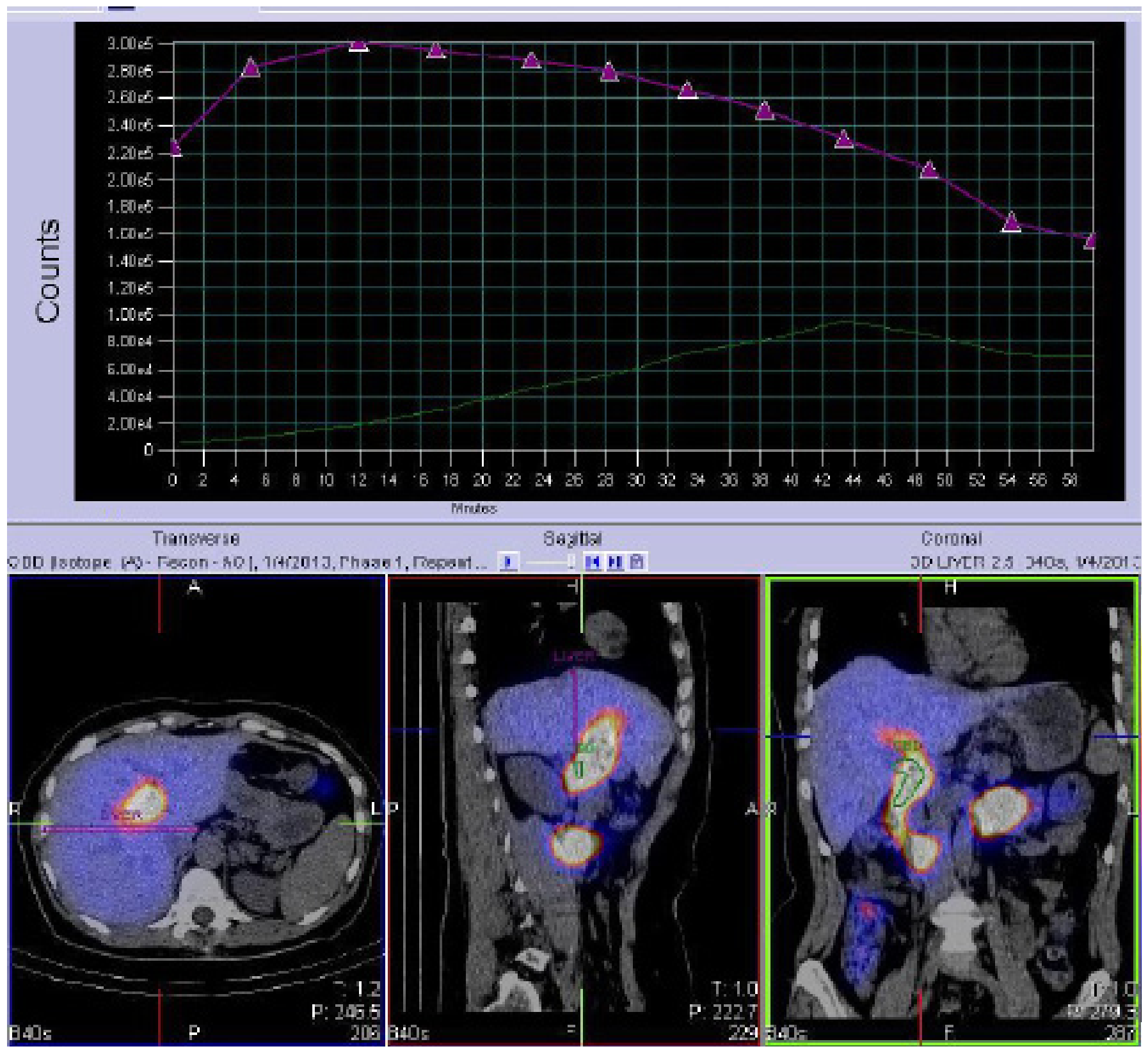

Figure 11: A (+) SOD patient with delayed clearance of bile from the liver and CBD 


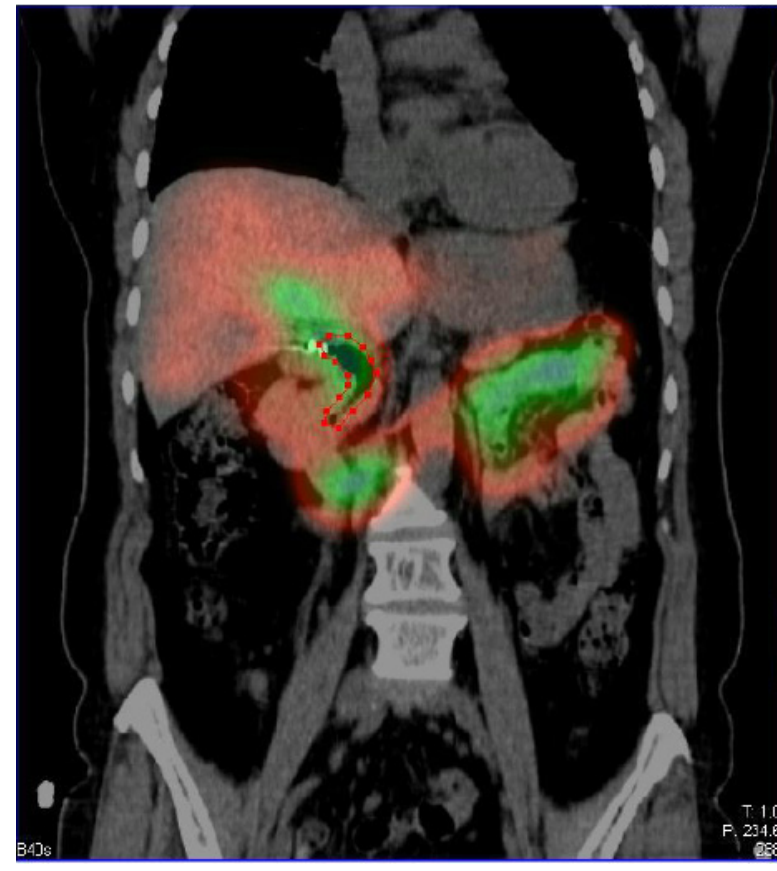

Figure 12: Red ROI around the CBD ends at duodenum

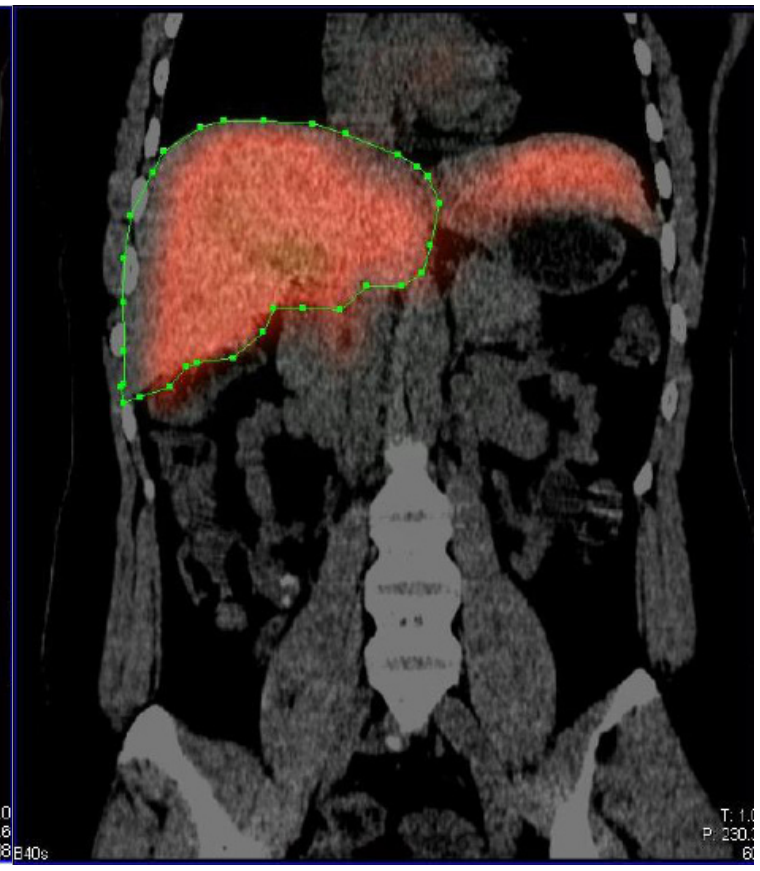

Figure 13: Green ROI around the RLL aviods intrahepatic ducts

Identification of the $\mathrm{CBD}$ and discriminating it form the duodenum is clearly much easier since the physician can scroll through multiple images to identify the beginning and end of each vital structure. Once a single ROI is drawn for the CBD and RLL, the TAC are generated automatically. Therefore, the ROI can be re-adjusted to precisely pinpoint the CBD and RLL, without having to manually regenerate another set of TACs.

Because drawing ROI is only done once for each structure and TACs are generated automatically, the processing of this study, has become much quicker. Experienced technologists were able to process SPECT/CT HIDA SOD scans in $<15$ minutes. Overlapping loops of bowel are no longer an issue. Identifying the transitions of the liver, CBD and duodenum is less cumbersome (Figure 12 and 13) [19], and finding the end of the common hepatic duct and the beginning of the CBD was much easier since often there is a metallic surgical clip to identify the site of the cholecystectomy (Figure 8 and 12) [19].

\section{Conclusion}

SOD is a fairly common in post cholecystectomy patients, but has been difficult to diagnose noninvasively. Hepatobiliary scintigraphy has been shown to be one of the most useful noninvasive methods of identifying SOD. The current study clearly demonstrates significantly slower bile kinetics through the liver and CBD of (+) SOD patients when compared with patient that have normal functioning sphincter of Oddi. Unfortunately, biliary scintigraphy has been underutilized for diagnosing SOD, because it is a 2-D imaging method that lacks anatomical details and requires long processing times as well as tedious data collection. Furthermore due to the small size of the CBD $(0.5$ to $1.0 \mathrm{~cm})$ and the difficulty of finding the junction between the CBD and the duodenum, drawing ROI on planar scintigraphy is difficult. By using SPECT/CT imaging, 3-D information about bile kinetics can be acquired and anatomical correlation is simultaneously available, thus increasing diagnostic ability and dramatically reducing processing time.

\section{References}

1. Maudar KK (2008) Sphincter of Oddi Dysfunction. Post-Graduate Medical J NAMS 8: 1-5.

2. Shaffer E, Curley M (2013) Biliary Dyspepsia: Functional Gallbladder and Sphincter of Oddi Disorders In: Dyspepsia - Advances in Understanding and Management 111-34.

3. Sostre S, Kalloo AN, Spiegler EJ, Camargo EE, Wagner HN Jr (1992) A noninvasive test of sphincter of Oddi dysfunction in postcholecystectomy patients: the scintigraphic score. J Nucl Med 33: 1216-22.

4. Sgouros SN, Pereira SP (2006) Systematic review: sphincter of Oddi dysfunction - non-invasive diagnostic methods and long-term outcome after endoscopic sphincterotomy. Aliment Pharmacol Ther 24: 237-46.

5. Symbia T (2012) Turnover Workbook. Siemens Medical Solutions, Inc.

6. Craig AG, Peter D, Saccone GT, Ziesing P, Wycherley A, et al. (2003) Scintigraphy versus manometry in patients with suspected biliary sphincter of Oddi dysfunction. Gut 52: 352-7.

7. Hogan WJ, Geenen JE (1988) Biliary dyskinesia. Endoscopy 20: 179-83.

8. Rolny P, Arlebäck A, Funch-Jensen P, Kruse A, Ravnsbaeck J, et al. (1986) Paradoxical response of sphincter of Oddi to intravenous injection of cholecystokinin or ceruletide. Manometric findings and results of treatment in biliary dyskinesia. Gut 27: 1507-11. 
9. Toouli J, Roberts-Thomson IC, Dent J, Lee J (1985) Manometric disorders in patients with suspected sphincter of Oddi dysfunction. Gastroenterology 88: 124350 .

10. Cicala M, Habib FI, Vavassori P, Pallotta N, Schillaci O, et al. (2012) Outcome of endoscopic sphincterotomy in post cholecystectomy patients with sphincter of Oddi dysfunction as predicted by manometry and quantitative choledochoscintigraphy. Gut 50: 665-8.

11. Fullarton GM, Hilditch T, Campbell A, Murray WR (1990) Clinical and Scintigraphic assessment of the role of endoscopic sphincterotomy in the treatment of sphincter of Oddi dysfunction. Gut 31: 231-5.

12. Thomas PD, Turner JG, Dobbs BR, Burt MJ, Chapman BA (2000) Use of 99mTc-DISIDA biliary scanning with morphine provocation for the detection of elevated sphincter of Oddi basal pressure. Gut 46: 838-41.

13. Tulchinsky M, Ciak BW, Delbeke D, Hilson A, Holes-Lewis KA, et al. (2010) SNM practice guideline for hepatobiliary scintigraphy 4.0. J Nucl Med Technol 38: $210-8$.

14. Madácsy L, Middelfart HV, Matzen P, Hojgaard L, Funch-Jensen P (2000) Quantitative hepatobiliary scintigraphy and endoscopic sphincter of Oddi manometry in patients with suspected sphincter of Oddi dysfunction: assessment of flow-pressure relationship in the biliary tract. Eur J Gastroenterol Hepatol 12: 777-86.

15. Thompson JC, Fender HR, Ramus NI, Villar HV, Rayford PL (1975) Cholecystokinin metabolism in man and dogs. Ann Surg 182: $496-504$.

16. Krishnamurthy GT, Krishnamurthy S (2000) Nuclear Hepatology: A Textbook of Hepatobiliary Diseases, Springer Science \& Business Media Publisher, USA.

17. Gonzalez AA, Farrea R, Moneas J, Capella G, Clave P (2000) Pharmacological and molecular characterization of muscular cholecystokinin receptors in the human lower oesophageal sphincter. Neurogastroenterol Mot 12: 539-46.

18. Rajesh Upadhyay (2010) Clinical Approach to Dilated Bile Duct. Medicine Update 20: 477-80.

19. Symbia T16/T6/T2/T (2014) Series Release Information-syngo MI Applications VB10.

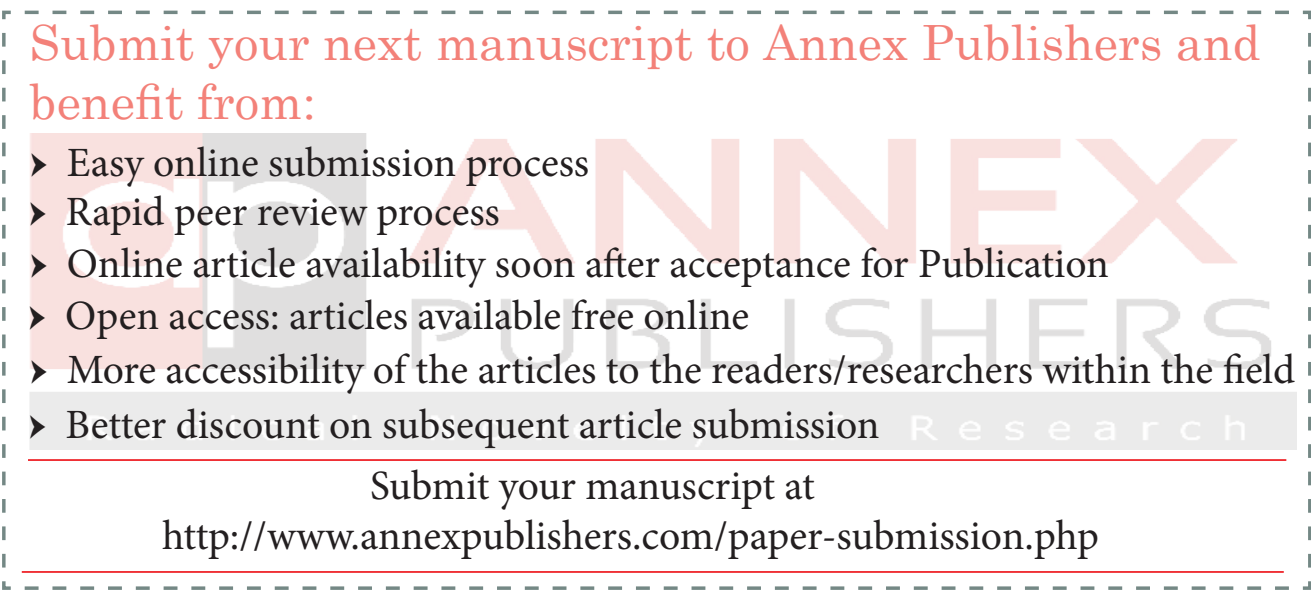

